\title{
Blend of Metal Oxides and Polyaniline on Platinum Electrodes for Dissolved Oxygen Sensors
}

\author{
Tien Minh Huynh ${ }^{1}$, Tung Son Vinh Nguyen ${ }^{2}$, Tin Chanh Duc Doan ${ }^{3}$ Chien Mau Dang ${ }^{4}$ \\ 1,2,3,4 Institute for Nanotechnology, Vietnam National University - Ho Chi Minh City, Community 6, Linh Trung Ward, Thu Duc \\ District, Ho Chi Minh City, Vietnam
}

\begin{abstract}
In this study, we report the detection of dissolve oxygen (DO) by using a blend of Ruthenium oxide and polyaniline (PANI) coated on platinum electrodes. Optical properties of the PANI were characterized by using Ultraviolet - Visible (UV-Vis) and Fourier-transform infrared (FTIR) spectroscopy. In order to study the sensitivity of the thin films of the PANI and PANI: Ruthenium oxide blend, cyclic voltammetry (CV) measurements was performed with the platinum electrodes coated with the thin films of PANI: Ruthenium oxide. The electrolyte solutions were prepared from the phosphate buffer and had the salinity of $20 \%$ and $\mathrm{pH}$ 7.3. The CV measurements were carried out when the electrodes were put in the electrolyte solutions which had various DO concentrations by purging pure nitrogen and oxygen gases. The DO concentrations in the experiments were measured by a commercial dissolved oxygen probe. The correlation between the current in the CV measurements and DO concentrations was determined. The effect of the blending ratio of the metal oxide and PANI on the electrochemical signal was also specified. The results showed that the platinum electrodes with PANI: Ruthenium oxide blend coating exhibited a high possibility as electrochemical sensor for detection of low levels of DO in aqueous phase.
\end{abstract}

KEYWORDS: Electrochemical sensors, Dissolved Oxygen, Polyaniline, Ruthenium oxide, Leuco Emeraldine Base Polyaniline (LEB-PANI).

\section{INTRODUCTION}

The determination of dissolved oxygen (DO) concentration is one of the most important parameter of water treatment, aquaculture and biological research. The amount of DO can directly affect the bacteria level of the water. Therefore, it is needed to be continuously monitored and controlled in order to have sufficient information for the quality of water.

Until now, detection of DO can be done by two major techniques such as Winkler method [1] and amperometric method [2]. The Winkler method which requires large amount of samples and numerous of steps is not suitable for the continuous measurements. On the other hand, the amperometric method which is so called Clark electrode, is an electrochemical method, can provide fast response and easy control. This method is referred as Clark electrochemical or membrane - covered electrode [3]. Most of the DO sensors on the market nowadays employ optical technologies. However, one of the disadvantages of these sensors is high price, which is a big drawback of their application in the industry field. Therefore, the demand of a compact DO sensor which has low cost, easy operation has been increased significantly and attracted the interest of scientists all over the world.

The optical methods have been used to measure DO concentrations in micro-sized water streams with octaethyporphrine capable of platinum-reducing oxygen (II) associated with thin film of polystyrene [4]. Another example is the report on the DO levels measured in a cell culture media base on the intensity of the fluorescence of dextran and ruthenium complex immobilized on poly(ethylene glycol) di-acrylate microarray [5]. However, the optical method is just suitable for the measurements in the laboratory and it is hard to apply in the field application. Besides, the electrochemical technology has been widely used for DO sensor development [6-8]. Chou and his colleague investigated gold/ Nafion electrodes with $\mathrm{H}_{2} \mathrm{SO}_{4}$ acid as a supporting electrolyte for detection of DO in solvent [7]. Peng Wang et al. [8] used a silicon microprobe including Nafion coating on three platinum electrodes to measure DO levels at $0 \%$ atm, $10.5 \%$ atm and $21 \%$ atm.

Recently, there have been many types of sensors using conductive polymers [9-17], but only a few of them have been applied for DO measurement. Tieman and his co-workers have measured DO levels by using ionically conductive polymer poly (dimethyldiallylammonium chloride) coated on graphite electrodes as working electrodes [18]. The authors used $\mathrm{Pt}$ auxilary electrode and $\mathrm{Ag} / \mathrm{AgCl}$ reference electrode. DO concentration was measured by reduction at the potential of $-0.40 \mathrm{~V}$ versus $\mathrm{Ag} / \mathrm{AgCl}$. The linearity of concentration was in the range of 1.4-9.3 ppm. Moreover, platinum electrode coated with polyaniline that was studied as a potentiometric sensor to

Tien Minh Huynh ${ }^{1}$, ETJ Volume 6 Issue 06 June 2021 
measure DO concentration [19]. This PANI-coated electrode gave oxygen concentrations of about $97 \mathrm{mV} /$ decade and a voltage signal of about $400 \mathrm{mV}$. The limitation of detection (LOD) of DO was estimated at $5 \times 10-7 \mathrm{~mol} / \mathrm{l}$. The proposed mechanism was based on redox oxidation of LEB- PANI which led to conversion into conducting salt PANI in the presence of oxygen bubbles in $\mathrm{H}_{2} \mathrm{SO}_{4}$ solution. In this study, the $\mathrm{pH}$ of the medium was kept below 7 with a mixture of $\mathrm{H}_{2} \mathrm{SO}_{4}$ and $\mathrm{NaOH}$. The doping of oxidation by existing $\mathrm{H}_{2} \mathrm{SO}_{4} 1 \mathrm{M}$ solution might have caused the transformation into the conducting salt form of PANI. Therefore, the oxidation mechanism proposed by oxygen aeration was still unclear.

Moreover, Ruthenium compounds are known as very attractive oxygen-sensing materials thanks to their long lifetime, strong absorption in the blue-green region of the UV-Vis spectrum, and large Stokes shift [20-24]. The dye of ruthenium compounds was entrapped into a microporous, hydrophobic sol-gel-derived cage-like microstructure that retained oxygen molecule permeability. When the dye reacted with oxygen, the fluorescence of the dye could be rapidly quenched. As the quenching time was proportional to the oxygen concentration, when the emitted light was modulated using a square signal, the phase shift of the fluorescence would also be proportional to the oxygen concentration. Hence the phase information of the fluorescence could be used to represent the oxygen concentration.

In this article, Leuco Emeraldine Base Polyaniline (LEB-PANI) was blended with metal oxides $\left(\mathrm{RuO}_{2}\right.$ and $\left.\mathrm{Cu}_{2} \mathrm{O}\right)$ and these blends were used to detect DO concentration in solution. UV-Vis and FTIR spectroscopy and Cyclic Voltammetry (CV) were used to characterize the materials and evaluate the DO sensitivity. The correlation of the current in the CV measurements and DO concentrations was studied and discussed.

\section{EXPERIMENT}

\subsection{Materials}

Emeraldine Base Polyaniline (EB-PANI) (99.7 \%, Mw 20000), dimethyl sulfoxide (DMSO) (99.9 \%), phenylhydrazine (PHZ) (97\%), hydrochloric acid ( $\mathrm{HCl})(37 \%)$ were perchased from Merck, Germany. $\mathrm{RuO}_{2}$ and $\mathrm{Cu}_{2} \mathrm{O}$ were bought from Disodium hydrogen phosphates dodecahydrate $\left(\mathrm{Na}_{2} \mathrm{HPO}_{4} \cdot 12 \mathrm{H}_{2} \mathrm{O}\right)$, sodium dihydrogen phosphate dihydrate $\left(\mathrm{NaH}_{2} \mathrm{PO}_{4} .2 \mathrm{H}_{2} \mathrm{O}\right)$ and sodium chloride $(\mathrm{NaCl})$ which were bought from Xilong, China were used to prepare the electrolyte solutions. All the chemicals were used as purchased without further purification.

Oxygen $\left(\mathrm{O}_{2}\right)$ gas $(99.99 \%)$ was supplied by Singapore Oxygen Air Liquide Pte. Ltd. Deionized (DI) water was supplied by a DI system (Purelab Ultra, Elga Co., UK), the resistance of the outlet water was 18.2 MOhms.

\subsection{Synthesis of polymer solutions}

\subsubsection{LEB-PANI preparation}

Mix $30 \mathrm{mg}$ of EB-PANI with $2.7 \mathrm{ml}$ DMSO, stirring for 24 hours with a magnetic stirrer at room temperature to obtain EB-PANI solution. The solution was then filtered through a filter paper. The obtained polymer solution had dark blue color. EB-PANI powder was protonated by $\mathrm{HCl} 0.1 \mathrm{M}$ solution to obtain ES-PANI (green color). The salt was filtered and rinsed by DI water to remove residual acid. Then ES-PANI was dried under vacuum at $60^{\circ} \mathrm{C}$ in a vacuum oven. $30 \mathrm{mg}$ ES-PANI was dispersed in $2.7 \mathrm{ml}$ DMSO to obtain ES-PANI solution. This solution underwent ultra-sonification and centrifugal mixing. ES-PANI was mixed with PHZ 97\% to obtain LEB-PANI. Then the powder was rinsed in DI water, acetone and methanol to remove residual $\mathrm{PHZ}$ and dried at $60^{\circ} \mathrm{C}$ in the vacuum oven. After that, LEB-PANI was dissolved in DMSO and this solution underwent ultra-sonification and centrifugal mixing.

\subsubsection{Blending PANI with metal oxides ( $\mathrm{Cu}_{2} \mathrm{O}$ and $\left.\mathrm{RuO} \mathrm{O}_{2}\right)$}

$30 \mathrm{mg}$ of $\mathrm{Cu}_{2} \mathrm{O}$ was well-mixed with $270 \mathrm{mg} \mathrm{RuO}$ (sintered at $1,000^{\circ} \mathrm{C}$ to stabilized the crystal structure) to form metal oxide mixture which contains $10 \%$ of $\mathrm{Cu}_{2} \mathrm{O}$ in order to increase the oxygen sensitive [25]. After that, $100 \mathrm{mg}$ of metal oxide mixture was mixed with LEB-PANI powder with the ratio of 1:2 and 1:3. Finally, all the powder was mixed with DMSO to form a solution which had concentration of $1 \%$. All solution was stirred for $24 \mathrm{~h}$.

\subsubsection{Coating the platinum electrodes with LEB-PANI: metal} oxides

Electrochemical chips included one working electrode and one counter electrode (dimension of $3 \mathrm{~mm}$ x $9 \mathrm{~mm}$ ) put separately with a distance of $3 \mathrm{~mm}$. Both electrodes were made of platinum $(\mathrm{Pt})$. Pt layers were sputtered with Univex 350 (Leybold, Germany) and patterned on silicon ( $\mathrm{Si}$ ) wafers coated with $700 \mathrm{~nm}$ thick $\mathrm{SiO}_{2}$ by photolithography and lift off techniques. The Pt electrodes had a thickness of $190 \mathrm{~nm}$ with a titanium adhesion layer.

$4 \mu \mathrm{l}$ of the LEB-PANI: metal oxide solutions were drop-casted on the working electrode of the electrochemical chips and these chips were dried in vacuum oven at $60^{\circ} \mathrm{C}$ for 24 hours to remove completely DMSO.

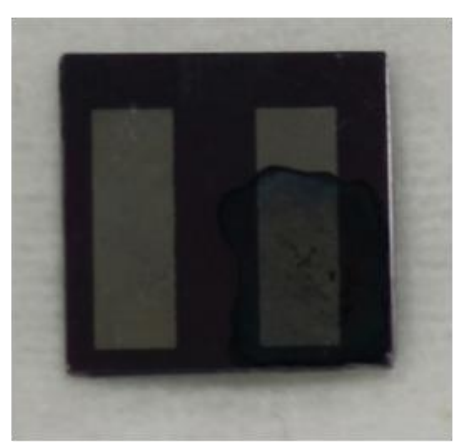

Figure 1. The platinum electrodes coated with the PANI blend film on the working electrode.

\subsection{Charaterization}

The obtained nanoparticles were characterized using X-ray 
diffractometer (XRD), ultraviolet-visible spectrometer (UV-Vis) (Cary 100), transmission electron microscope (TEM) (JEM model $1400,100 \mathrm{kV}$ ). Particle size distribution was obtained using ImageJ software.

The PANI was characterized by UV-Vis spectroscopy (Cary 100 Varian, USA) and Fourier-transform infrared (FTIR) spectroscopy. The thickness of the Pt electrodes and the blend films after fabrication was measured by a stylus profiler Dektak $6 \mathrm{M}$ (Veeco, USA). The $\mathrm{RuO}_{2}$ before and after heat-treatment was characterized with X-Ray Diffraction (XRD).

\subsection{Potentiometric measurements}

The testing electrolyte solutions were prepared from phosphate buffer which was made from $6 \mathrm{~g} \mathrm{Na}_{2} \mathrm{HPO}_{4} .12 \mathrm{H}_{2} \mathrm{O}$, $0.5 \mathrm{~g} \mathrm{NaH}_{2} \mathrm{PO}_{4} .2 \mathrm{H}_{2} \mathrm{O}$ and DI water to obtain $200 \mathrm{ml}$ testing solution with $\mathrm{pH}$ 7.3. Then, $4 \mathrm{~g} \mathrm{NaCl}$ powder was added to make the solution have salinity of $20 \%$. The testing solution was prepared to imitate the conditions of aquaculture water.

The testing solutions were bubbled with nitrogen $\left(\mathrm{N}_{2}\right)$ gas for 15 minutes to minimize DO concentration in the solution, approximately $1.5 \mathrm{ppm}$. Then, oxygen $\left(\mathrm{O}_{2}\right)$ gas was bubbled through the solution with a pressure of 1 psi for 15-90 seconds to create various DO concentrations in the solutions. DO concentrations were measured by a commercial DO probe D06400TC/T (Sensorex, US) with a measurement range of $0-20 \mathrm{mg} / 1,0.01 \mathrm{mg} / 1$ resolution and $\pm 1.5 \%$ accuracy.

The blend coated chips were dipped in the testing solutions in which the layer part was immersed completely. The CV measurements were performed with a potentiostat/galvanostat PGSTAT 302N (Metrohm, Autolab, the Netherlands) and a silver/silver chloride $(\mathrm{Ag} / \mathrm{AgCl})$ reference electrode RE-6 (Basi Inc., US). The blend chips were mounted on two crocodile clips (Fig. 2). The current was set from $10 \mu \mathrm{A}$ to $10 \mathrm{~mA}$, the scanning rate was $50 \mathrm{mV} / \mathrm{sec}$, the temperature was about $28^{\circ} \mathrm{C}$ $\pm 1{ }^{\circ} \mathrm{C}$.

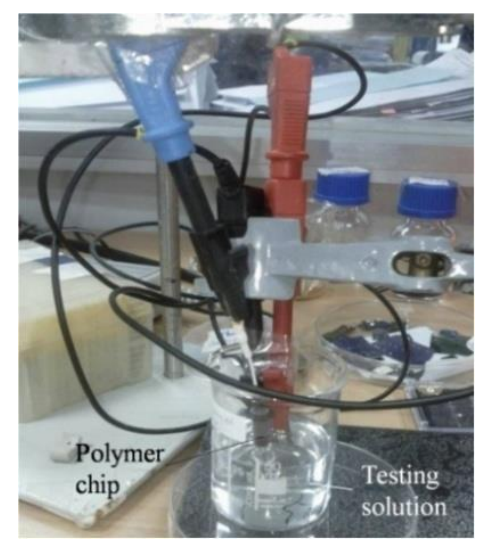

Figure 2. The blend chip mounted on two crocodile clips.

\section{RESULTS AND DISCUSSION}

\subsection{UV-Vis spectra of the PANI solutions}

Fig. 3 showed that the UV-Vis spectrum of EB-PANI in DMSO solvent shows two peaks at wavelength of $324 \mathrm{~nm}$ and $625 \mathrm{~nm}$. This result is in good agreement with literature values [26-28]. The absorption peak at $324 \mathrm{~nm}$ is assigned to the $\pi$ $\pi^{*}$ transition of the benzenoid rings. The peak at $625 \mathrm{~nm}$ originates from the exciton transition of the quinoid rings [27]. The absorption spectrum of $\mathrm{HCl}$-doped PANI in DMSO solvent shows peaks at $289 \mathrm{~nm}, 456 \mathrm{~nm}$ and $663 \mathrm{~nm}$. The two bands were assigned to the optical absorption of the polaron band [26, 28]. The absorption spectrum of LEB-PANI has only one peak at $292 \mathrm{~nm}$, which is in good agreement with the published value [29].

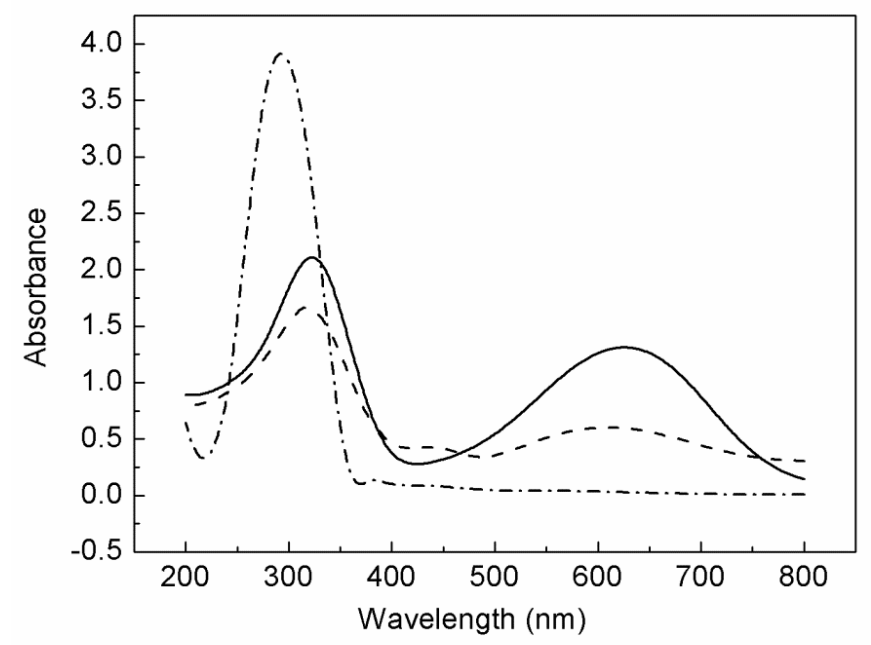

Figure 3. UV -Vis absorption spectra of EB-PANI (solid line), ES-PANI (dash line) and LEB-PANI (dash dot line) in DMSO.

\subsection{FTIR spectra of LEB-PANI}

FTIR spectra of the LEB-PANI prepared from the experimental section and the LEB-PANI from the commercial are presented in Fig. 4. These results are in good agreement with the published reports by Hwang et al. [30], Brozova et al. [31] and Choi et al. [32]. Based on the spectra, it showed the stretching bands of the benzenoid and quinoid rings of the PANI which are determined at $1,593 \mathrm{~cm}^{-1}$ and $1,494 \mathrm{~cm}^{-1}$, respectively. The peak at 1,284 is indicated to the stretching mode of the imine group $(\mathrm{C}=\mathrm{N})$. The electronic-like absorption of the $\mathrm{N}=\mathrm{Q}=\mathrm{N}$ vibration (where $\mathrm{Q}$ is specified the out of plane bending vibration of the para- substituted benzene ring) has appeared at $1,154 \mathrm{~cm}^{-1}$. The FTIR results showed that the LEB-PANI was well-prepared with high purity, compared to the commercial product. 
"Blend of Metal Oxides and Polyaniline on Platinum Electrodes for Dissolved Oxygen Sensors"

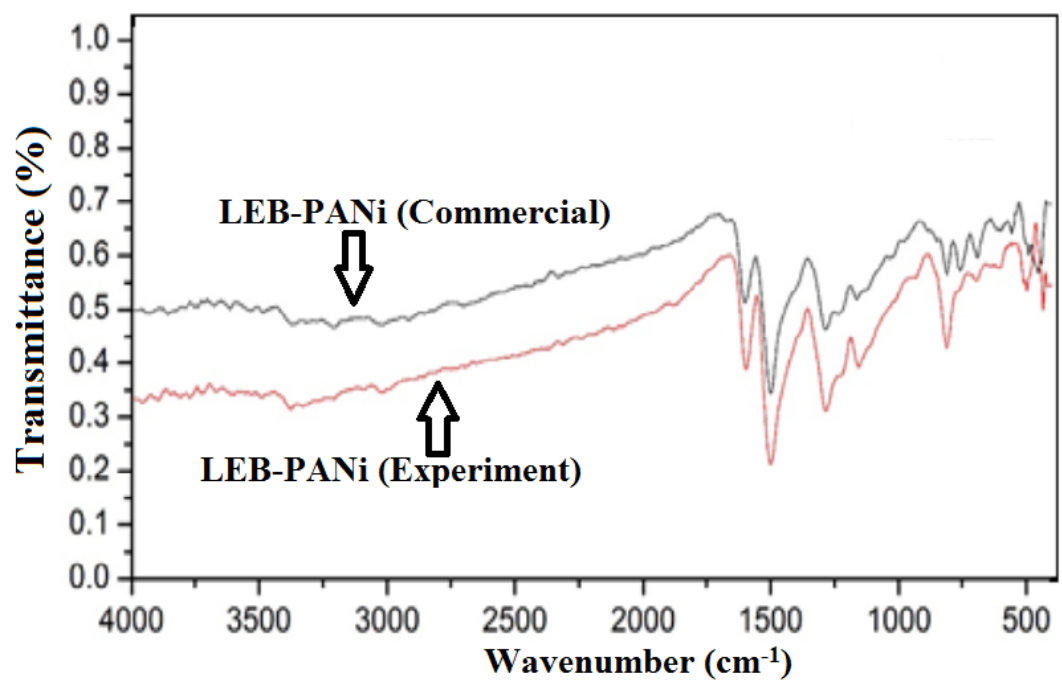

Figure 4. The FTIR spectra of the LEB-PANI experiment compared with the commercial LEB-PANI.
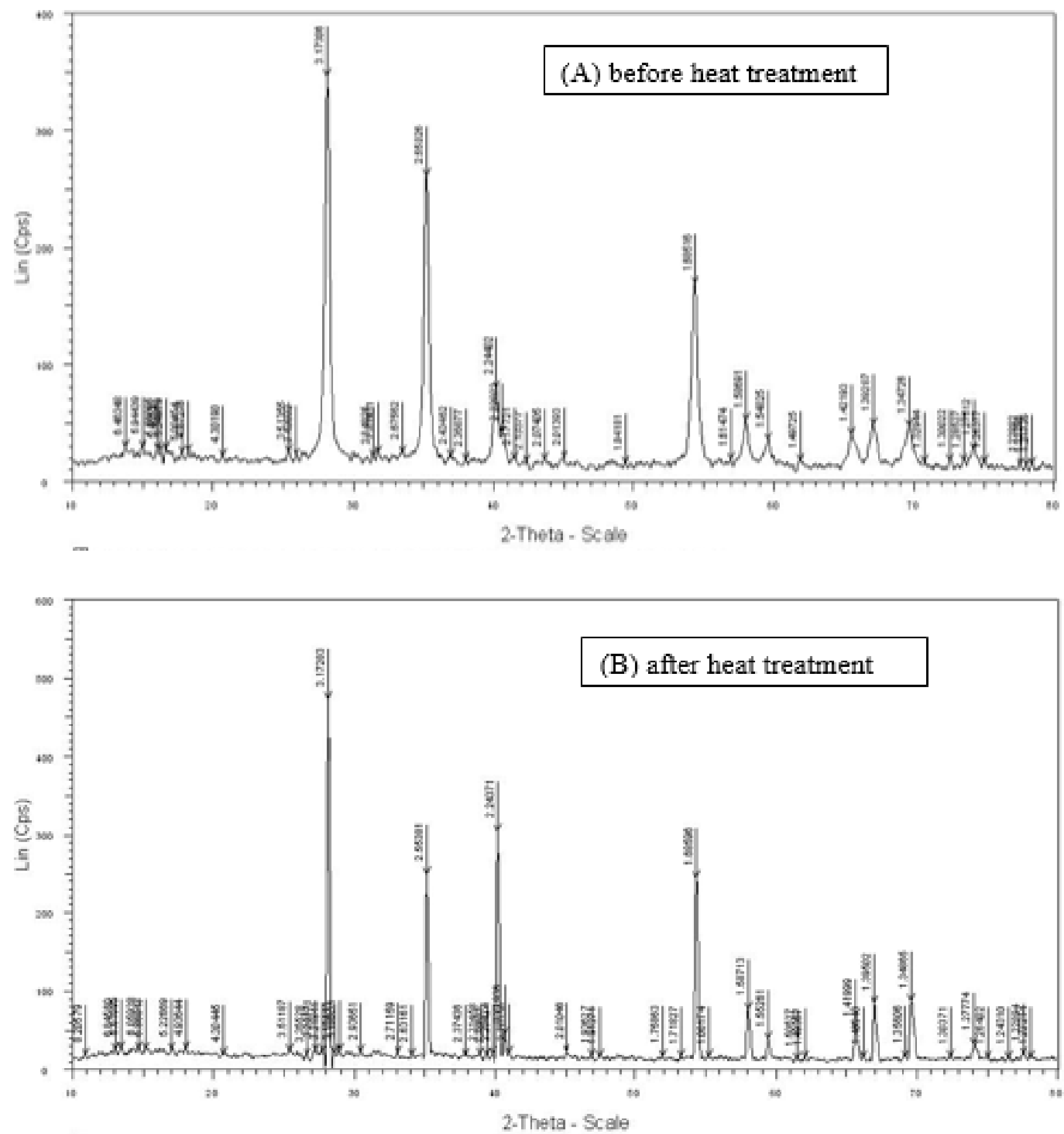

Figure 5. The XRD patterns of the $\mathrm{RuO}_{2}$ powder before (A) and after heat treatment (B).

\subsection{XRD patterns of $\mathrm{RuO}_{2}$}

The $\mathrm{RuO}_{2}$ powder was heat-treated at $1,000^{\circ} \mathrm{C}$ to stabilize the crystal structure and create stability of the metal oxide. The XRD characterization results of the oxide powder before and after heating were presented in the Fig. 5. The diffraction peaks 
at $28^{\circ}, 35^{\circ}, 40^{\circ}, 54^{\circ}$ showed the quadrilateral structure of $\mathrm{RuO}_{2}$ as in the report of Serge Zhuiykov [25]. The results showed that after high-temperature treatment, the intensity of diffraction peaks increased, it meant that the crystallinity of the oxide powder increased correspondingly.

\subsection{CV measurements of the blend-coated electrodes}

Fig. 6 shows the CV plots of the LEB-PANI-coated chips upon exposure with various electrolyte solutions that had different DO levels. It showed one reduction peak at $-0.03 \mathrm{~V}$ which was similar with the result from Edward Song et al. [33].

There was a significant increase in the current when DO concentration increased from $1.5 \mathrm{ppm}$ (corresponding to $\mathrm{N}_{2}$ purging) to $26 \mathrm{ppm}$ (1.5 minutes of oxygen bubbling). The current increased with DO concentrations from $0.018 \mathrm{~mA}$ to $0.1 \mathrm{~mA}$ at $-0.03 \mathrm{~V}$. Fig. 7 shows the relationship between the cathode current at $-0.03 \mathrm{~V}$ and the DO concentrations. The increase in current of the LEB-PANI thin films followed an exponential Decay function. The current of the LEB-PANI films increased steeply from $1.5 \mathrm{ppm}$ to $10 \mathrm{ppm}$ DO and did not change much after $15 \mathrm{ppm}$ DO. The change in the current corresponding to estimated DO concentration was $1.18 \mu \mathrm{A} /$ ppm of the DO concentration.

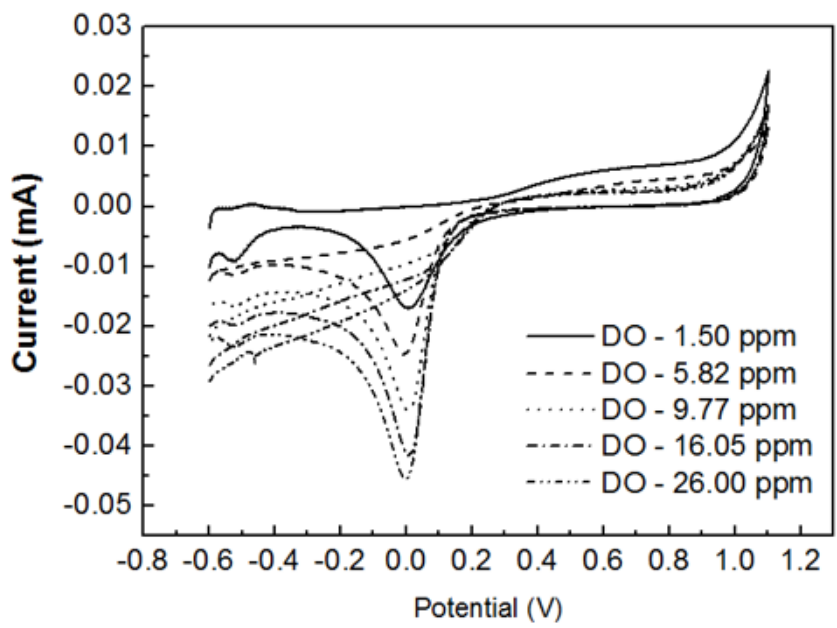

Figure 6. CV plots of LEB-PANI-coated chips with different DO concentrations.

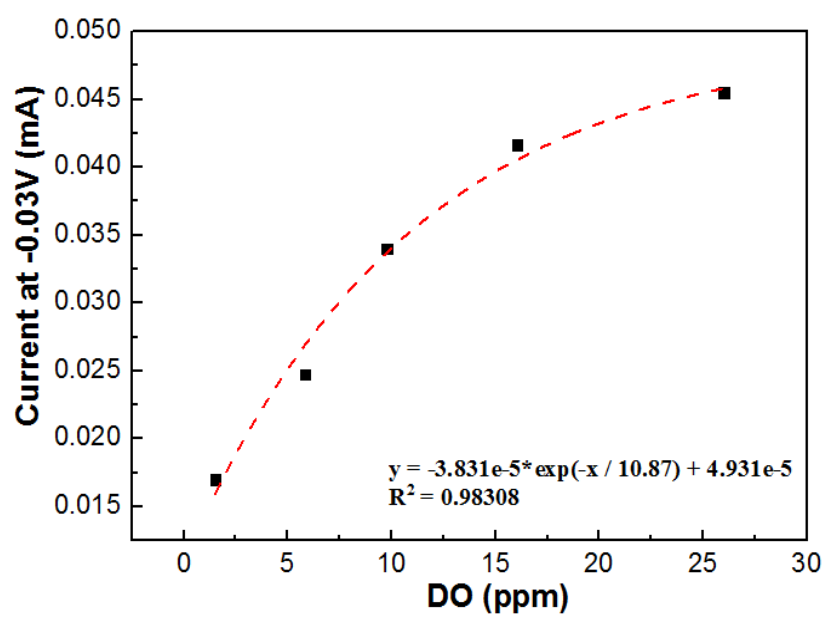

Figure 7. The relationship between the cathode current and DO concentrations at $-0.03 \mathrm{~V}$ of the LEB-PANI coated chip.
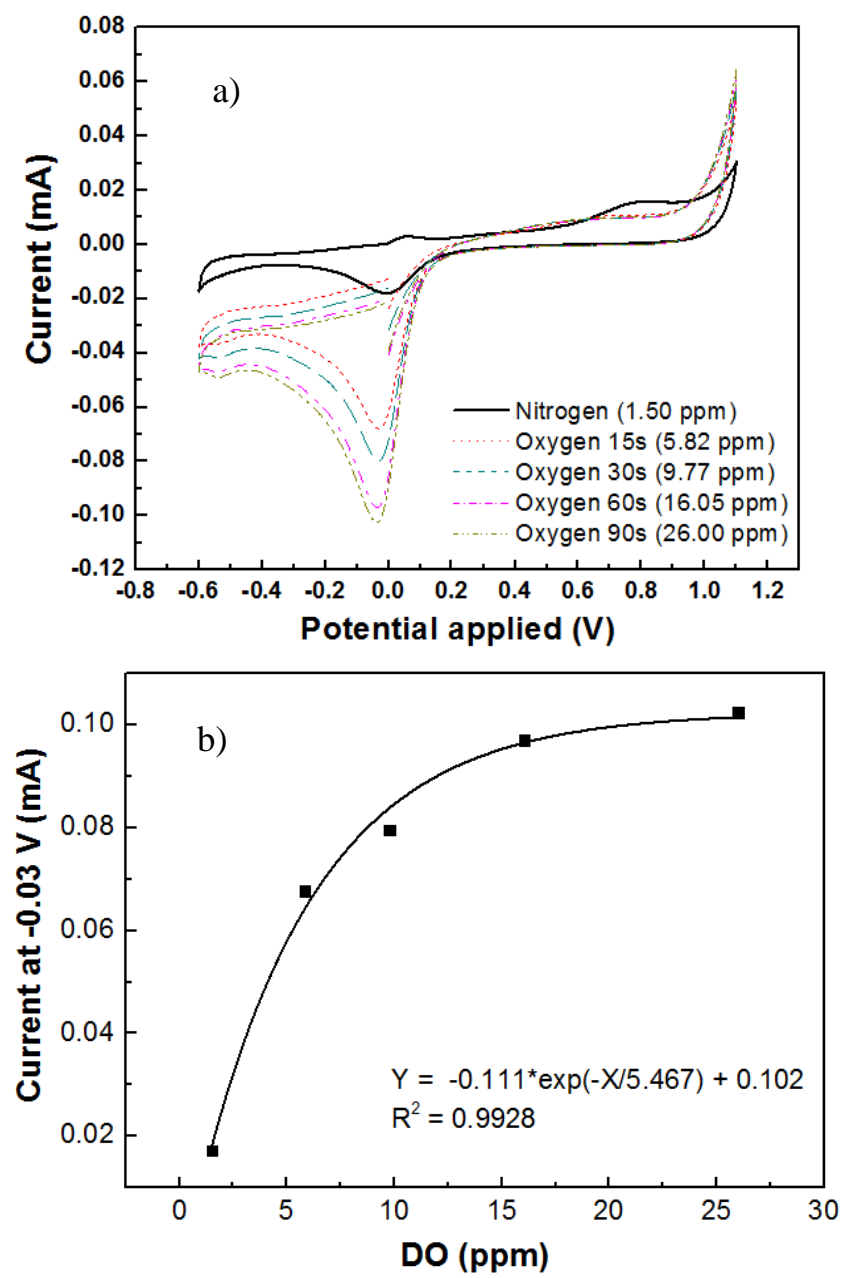

Figure 8. $\mathrm{CV}$ plots of the blend with ratio of 1:2 chips upon exposure with various DO concentrations (a), the fitting curves of the cathode peak intensity with DO concentrations (b).

Fig. 8 shows the CV plots of the blend of LEB-PANI: metal oxide with the ratio of 1:2 for metal oxide:LEB-PANI. Similar to the result of the LEB-PANI chips, the $\mathrm{CV}$ had one reduction peak at $-0.03 \mathrm{~V}$. When the DO concentration increased, it led to the increase in the magnitude of the current went through the surface of the electrode. The addition of the metal oxide increased the correlation coefficient of the relation between the current and the DO concentration (from 0.98308 to 0.9928). The increase in current as the function of the change in DO concentrations was $3.48 \mu \mathrm{A} / \mathrm{ppm}$. Hence, it could be concluded that the blend of the metal oxide with LEB-PANI increased the DO sensitivity of the fabricated electrode.

\section{CONCLUSION}

In this article, we reported the possibility of using the blend of LEB-PANI and metal oxides for detection of the DO concentrations in aqueous media. With the presence of the $\mathrm{RuO}_{2}$ mixed with $10 \%$ of $\mathrm{Cu}_{2} \mathrm{O}$, the increase in the current of the blend-coated chips as the function of the change in DO concentrations was higher than the current increase of the LEB-PANI coated chips. Therefore, the using of the blend of LEB-PANI and metal oxides could be promising to enhance the sensitivity for DO electrochemical sensors.

Tien Minh Huynh", ETJ Volume 6 Issue 06 June 2021 


\section{Acknowledgements}

The authors highly appreciate the financial support of First Project under the grant number 09/FIRST/2a/INT.

\section{REFERENCES}

1. H. Culberson, C., et al., A comparison of methods for the determination of dissolved oxygen in seawater. 2019.

2. Chen, G., Electrochemical Technologies in Wastewater Treatment Separation. Vol. 38, 11-41, 2004.

3. Ibanez, J., et al., Dissolved Oxygen in Water. 2008.

4. Passaro, V.M.N., et al., Recent advances in integrated photonic sensors. Sensors (Basel, Switzerland), 12(11):15558-15598, 2012.

5. Lee, S., et al., Measurement of $\mathrm{pH}$ and dissolved oxygen within cell culture media using a hydrogel microarray sensor. Sensors and Actuators B: Chemical, 128(2): 388-398, 2008.

6. Fang, Y., et al., Fabrication of a Needle Microsensor and Its Applications in the Detection of Dissolved Oxygen. Journal of Sensors, 2015: 7, 2015.

7. Chou, T.C., K.M. Ng, and S.H. Wang, Gold-solid polymer electrolyte sensor for detecting dissolved oxygen in water. Sensors and Actuators B: Chemical, 66(1): 184-186, 2000.

8. Wang, P., et al., Micromachined dissolved oxygen sensor based on solid polymer electrolyte. Sensors and Actuators B: Chemical, 153(1): 145-151, 2011.

9. Bartlett, P.N. and P.R. Birkin, The application of conducting polymers in biosensors. Synthetic Metals, 61(1): 15-21, 1993.

10. Partridge, A.C., M.L. Jansen, and W.M. Arnold, Conducting polymer-based sensors. Materials Science and Engineering: C, 12(1): 37-42, 2000.

11. Nicolas-Debarnot, D. and F. Poncin-Epaillard, Polyaniline as a new sensitive layer for gas sensors. Analytica Chimica Acta, 475(1): 1-15, 2003.

12. James, D., et al., Chemical Sensors for Electronic Nose Systems. Vol. 149, 1-17, 2005.

13. Bai, H. and G. Shi, Gas Sensors Based on Conducting Polymers. Sensors (Basel, Switzerland), 7(3): 267-307, 2007.

14. Lange, U., N. Roznyatovskaya, and V. Mirsky, Conducting polymers in chemical sensors and arrays. Vol. 614, 1-26, 2008.

15. Liu, J., M. Agarwal, and K. Varahramyan, Glucose sensor based on organic thin film transistor using glucose oxidase and conducting polymer. Sensors and Actuators B: Chemical, 135(1): 195-199, 2008.

16. B Tolani, S., et al., Towards biosensors based on conducting polymer nanowires. Vol. 393. 1225-31, 2009.

17. Doan, T.C.D., et al., Carbon dioxide sensing with sulfonated polyaniline. Sensors and Actuators B:
Chemical, 168: 123-130, 2012.

18. Tieman, R.S., et al., Oxygen sensors based on the ionically conductive polymer poly (dimethyldiallylammonium chloride). Sensors and Actuators B: Chemical, 8(2): 199-204, 1992.

19. Shim, Y.-B., D. E. Stilwell, and S.-M. Park, Electrochemistry of conductive polymer $\mathrm{X}$ : Polyaniline-based potentiometric sensor for dissolved oxygen. Vol. 3, 31-36, 1991.

20. Xiong, X., D. Xiao, and M. M.F. Choi, Dissolved oxygen sensor based on fluorescence quenching of oxygen-sensitive ruthenium complex immobilized on silica-Ni-P composite coating. Vol. 117, 172-176, 2006.

21. Chu, F., et al., Characterization of a dissolved oxygen sensor made of plastic optical fiber coated with ruthenium-incorporated solgel. Vol. 48, 338-42, 2009.

22. Chan, M.A., et al., Fiber optic oxygen sensor based on phosphorescence quenching of erythrosin B trapped in silica-gel glasses. Analytica Chimica Acta, 408(1): 33-37, 2000.

23. Chan, M.A., et al., Fiber optic oxygen sensor based on phosphorescence quenching of erythrosin B trapped in silica-gel glasses. Vol. 408, 33-37, 2000.

24. Chuang, H. and M. A Arnold, Linear calibration function for optical oxygen sensors based on quenching of ruthenium fluorescence. Vol. 368. 1997.

25. Zhuiykov, S., et al., Potentiometric solid-state sensor for DO measurement in water using sub-micron $\mathrm{Cu}_{0.4} \mathrm{Ru}_{3.4} \mathrm{O}_{7}{ }^{+} \mathrm{RuO}_{2}$ sensing electrode. Sensors and Actuators B: Chemical, 153(2): 312-320, 2011.

26. Huang, W.S. and A.G. MacDiarmid, Optical properties of polyaniline. Polymer, 34(9): 1833-1845, 1993.

27. Hua, M.-Y., et al., Soluble n-Doped Polyaniline: Synthesis and Characterization. Macromolecules, 33(17): 6235-6238, 2000.

28. Shimano, J.Y. and A.G. MacDiarmid, Polyaniline, a dynamic block copolymer: key to attaining its intrinsic conductivity? Synthetic Metals, 123(2): 251-262, 2001.

29. P. McCall, R., et al., Spectroscopy and defect states in polyaniline. Vol. 41. 1990.

30. Hwang, G.-W., et al., Structures and properties of the soluble polyanilines, $\mathrm{N}$-alkylated emeraldine bases. Synthetic Metals, 92(1): 39-46, 1998.

31. Brozová, L., et al., The stability of polyaniline in strongly alkaline or acidic aqueous media. Vol. 93, 592-600, 2008.

32. Choi, C. and M. Kertesz, Conformational Studies of Vibrational Properties and Electronic States of Leucoemeraldine Base and Its Oligomers. Vol. 30. 1997.

33. Song, E. and J.-W. Choi, Conducting Polyaniline Nanowire and Its Applications in Chemiresistive Sensing. Vol. 3 498-523, 2013. 